ORIGINAL ARTICLE

\title{
Clinico-Pathological Patterns of Patients Who Underwent Orbital Exenteration in a Tertiary Eye Hospital of Nepal
}

\author{
Limbu Ben ${ }^{1}$, Mengesha Aemero², Anya G Gushchin ${ }^{3}$, Grant H Moore ${ }^{4}$, Saiju \\ Rohit $^{1}$
}

ABSTRACT

BACKGROUND: Orbital exenteration is a destructive procedure performed by removing all or part of the orbital contents along with entire eyeball. It is a procedure reserved for life threatening malignancies and some nonmalignant disorders which are not controlled by conservative management.

METHODS: This is a retrospective study done on patients who underwent orbital exenteration at Tilganga Institute of Ophthalmology from1 January 2006- 30 in December 2014.

RESULTS: The mean age of patients was 30 years (range 1-78), with male preponderance of 15(55.6\%. Overall presenting duration of eye morbidity was 18 months (2 months-8.5 years). The most common presenting complaint was protrusion of eyeball1, 4(50\%), primary site of tumor being intraocular in 10 patients $(35.7 \%$,) and total orbital exenteration was the most commonly performed type of surgery in 16(57.1\%) out of 27 patients. The most common etiology responsible for orbital exenteration, in pediatric age group of 9/27 patients (64.2\%), was retinoblastoma whereas conjunctival squamous cell carcinoma (SCC)accounts for 5 patients (38.4\%) in adults. Overall, the most common cause of orbital exenteration was retinoblastoma $9(32.1 \%)$.

CONCLUSION: The most common etiologies requiring orbital exenteration were retinoblastoma (in children and overall) and conjunctival squamous cell carcinoma (in adults), both diseases that could be addressed with less invasive treatment modality if detected earlier in the disease process. Designing strategy is important for early detection and treatment of these conditions, which would decrease disease morbidity and prognosis, potentially sparing sight and life.

KEYWORDS: Orbital exenteration, histopathology patterns, retinoblastoma, squamous cell carcinoma, proptosis, Nepal

DOI: http://dx.doi.org/10.4314/ejhs.v26i6.6

\section{INTRODUCTION}

Orbital exenteration, first described by George Bartisch in 1583(1), is a destructive procedure performed by removing all or part of the orbital contents along with the entire eyeball. Exenteration is performed when tumors of ocular surface, eyelid, lacrimal gland or intraocular tumor invade orbital tissues, in order to control the progression of the tumor from the orbit to the surrounding tissues and to distant organs. In addition, benign lesions causing massive proptosis, uncontrolled recurrent bleeding and severe uncontrolled infections may warrant exenteration (2-6).

In literature review, the common indications for exenteration were found to be eyelid malignancy and orbital tumor $(2,3,4,7)$. According to geographical locations, reports on common etiologies requiring exenteration variy Rahman et al (3), Wong et al (8) from the United Kingdom and Duman et al (4) from Turkey reported basal cell carcinoma as theleading pathology requiring exenterationwhereas Nemet et al from Australia reported Squamous cell carcinomaas as the most common cause (5).

\footnotetext{
${ }^{1}$ Tilganga Institute Of Ophthalmology, Nepal

${ }^{2}$ Department of Ophthalmology, JimmaUniversity, Ethiopia

${ }^{3}$ Moran Eye Center, University of Utah, USA

${ }^{4}$ Jules Stein Eye Institute, USA

Corresponding Author: Limbu Ben , Email: ben_limbu007@yahoo.com
} 
To our knowledge, there is no data on the clinicopathological pattern of exenteration in Nepal. The aim of the current study is to investigate the magnitude, presenting symptoms, indications and histopathology findings of patients who underwent orbital exenteration at a tertiary referral eye hospital in Kathmandu, Nepal.

\section{METHODS AND SUBJECTS}

This is a retrospective study conducted on patients who underwent orbital exenteration from 1 January 2006 to 30 December 2014, at Tilganga Institute of Ophthalmology, Nepal. The study was approved by the Institutional Review Board of Tilganga Institute of Ophthalmology.

All surgeries were performed under general anesthesia, and orbital exenteration was classified into three types(2).

1. Subtotal exenteration which was done by removing the globe for tumors involving the globe and conjunctiva and orbital contents but sparing the orbital periosteum and all or part of the eye lid.

2. Total exenteration which was done by removing all orbital contents along with the periosteum and eye lid; and

3. Eextended exenteration which was done by removing the orbital contents, periosteum, eye lid, and part of the orbital bone and paranasal sinus tissue

Each patient's medical records were reviewed to determine the presenting signs and symptoms, duration of symptoms prior to presentation, visual acuity at presentation, primary location of pathological lesions, indications for exenteration, clinical diagnosis, exenteration type and finally histopathology diagnosis. Surgical approach and adjunctive postoperative management were also reviewed.

A total of 31 eyes of 30 patients who underwent exenteration were identified and reviewed. Three of the 31eyes were excluded because of incomplete data documentation. Twenty-eight eyes of 27 patients were finally allocated for analysis. Statistical calculation was performed using Microsoft Excel 2011 version 14.4.8.

\section{RESULT}

The mean age of the study subjects was 30 years (ranging: 1-78 years) with 14/27 (52\%) patients being children of less than 15 years old. The majority of the patients were male, 15/27 (55.6\%). The most common presenting complaints were proptosis, $14 / 27(50.0 \%)$, followed by ocular surface growth, 5/27(17.9\%), and decreased vision, 4/27(14\%). The mean duration of symptoms prior to presentation was 18 months (range: 2 months -8.5 years), and majority of the patients presented within $\leq$ one month $15(53.6 \%)$. A right eye preponderance, 17/27(63.0\%), was also noted, and one patient had both eyes affected by orbital retinoblastoma (Table 1).

The most common primary anatomical site of pathology responsible for exenteration was intraocular, 10(35.7\%), followed by orbit, $8(28.6 \%)$. The most common surgical approach was subtotal exenteration ,11(64.71\%) (Table 2). 
Table1: Socio-demographic and clinical characteristics of orbital exenteration study patients.

\begin{tabular}{llc}
\hline Variables & Number of Participants (n=27) & Percentage (\%) \\
\hline Age in years & & \\
$\leq 15$ & 14 & 51.9 \\
$>15$ & 13 & 48.1 \\
Sex & Number of Participants (n=27) & \\
\hline Male & 15 & 55.5 \\
Female & 12 & 44.5 \\
Presenting chief complaint & Number of Eyes $(\mathbf{n = 2 8})$ & \\
\hline Protrusion of eyeball & 14 & 50 \\
Ocular Surface growth & 5 & 17.9 \\
Decreased vision & 4 & 14.3 \\
Eyelid mass & 3 & 10.7 \\
Leucocoria & 2 & 7.1 \\
Duration of presenting complaint & Number of Eyes $(\mathbf{n}=\mathbf{2 8})$ & \\
\hline$\leq 1$ month & 15 & 53.6 \\
1 month -1 year & 8 & 28.6 \\
$>1$ year & 5 & 17.8 \\
Laterality of Surgery & Number of Participants $(\mathbf{n}=\mathbf{2 7})$ & \\
\hline Unilateral & 26 & 96.3 \\
Bilateral & 1 & 3.7 \\
Affected eye & Number of Participants $(\mathbf{n}=\mathbf{2 7})$ & \\
\hline Right & 17 & 63.0 \\
Left & 9 & 33.3 \\
Both & 1 & 3.7 \\
\hline
\end{tabular}

We found that a large proportion of study patients had never consulted an eye health care facility, 9/27 (33\%) patients, and of the remaining 18 patients who visited eye care facility, 12/27 (44\%) had received some form of treatment prior to exenteration. Among the 12 patients who had prior intervention, 10 of the 12 patients received chemotherapy ( 3 patients for retinoblastoma and 6 topical chemotherapy). Among the 10 patients who received chemotherapy, one had received additional endolaser for retinoblastoma and another one had undergone enucleation for retinoblastoma. Out of two patients who did not receive chemotherapy before exenteration, one underwent incisional biopsy and the other had undergone enucleation prior to exenteration.

Histopathology demonstrated malignant tumors in the majority of our study patients, except in one patient where it showed a benign cystic lymphangioma. Overall, the most frequent malignant tumors identified were retinoblastoma, 9/27(32.1\%), conjunctival squamous cell carcinoma, 5/27 (17.9\%) and basal cell carcinoma of eyelid $3 / 27(10.7 \%$ ) (Table 3 ).

In $17 / 28(60.7 \%)$ of the cases, the surgical margin was infiltrated by tumor for which the patients were referred to an oncologist for further evaluation and management (Table 3). 
Table 2: Primary site of tumor versus types of exenteration $(n=28)$

Primary site of tumor

\begin{tabular}{llllc}
\cline { 2 - 5 } & Total & Subtotal & Extended & Total \\
& $\mathrm{N}(\%)$ & $\mathrm{N}(\%)$ & $\mathrm{N}(\%)$ & $\mathrm{N}(\%)$ \\
\hline Intraocular & $5(17.9)$ & $4(14.3)$ & $1(3.6)$ & $10(35.7)$ \\
Orbit & $4(14.3)$ & $4(10.7)$ & - & $8(28.6)$ \\
Ocular surface & $4(14.3)$ & $2(7.2)$ & - & $6(21.4)$ \\
Eye lid & $3(10.7)$ & $1(3.6)$ & - & $4(14.3)$ \\
Total & $16(57.1)$ & $11(39.3)$ & $1(3.6)$ & $28(100.0)$ \\
& & & & \\
\hline
\end{tabular}

Table 3: Histopathology reports and surgical margin involvement pattern among patients undergone exenteration in the study $(n=28)$.

\begin{tabular}{lll}
\hline Variables & No. & \% \\
\hline Histopathology diagnosis & 9 & 32.1 \\
Retinoblastoma & 5 & 17.9 \\
Conjunctiva squamous cell carcinoma & 2 & 7.1 \\
Non-Hodgkin's lymphoma & 2 & 7.1 \\
Malignant eye lid melanoma & 2 & 7.1 \\
Rhabdomyosarcoma & 1 & 3.6 \\
Basal cell carcinoma & 1 & 3.6 \\
olfactory neuroblastoma & 1 & 3.6 \\
Chroidal melanoma & 1 & 3.6 \\
Angiosarcoma & 1 & 3.6 \\
Malignant fibrous histiocytoma & 1 & 3.6 \\
Benign cystic lymphangioma & & \\
Surgical margin & 23 & 82.1 \\
Involved with the tumor & 3 & 10.7 \\
Not involved with the tumor & 2 & 7.1 \\
Unknown & & \\
\hline
\end{tabular}

In this study, we found that orbital retinoblastoma was the most common indication of exenteration in children ( $\leq 15$ years old) while conjunctival squamous cell carcinoma was the most common indication in adults ( $>15$ years old), accounting for $9 / 14(64.2 \%)$ and $5 / 13(38.5 \%)$ cases, respectively (Table 4).

\section{DISCUSSION}

Exenteration is a procedure reserved for life threatening malignancies. It is done for some nonmalignant disorders that are not controlled by conservative management.

The most common presentation was found to be proptosis in 14/28 cases (50\%) which was consistent with results of Kauret et al previous report (9). The most common indication for exenteration in our study was retinoblastoma $(32.1 \%)$, and this was discordant with other literature findings $(34,8,9)$ which reported that eyelid tumor was the most common etiology responsible for exenteration. This could be due to the distribution of patient age group in our study, consisting equal number of children and adults with high occurrence of retinoblastoma responsible for exenteration in childhood. We observed orbital tumor as the second most common primary site of tumor responsible for exenteration, which is similar with the findings of other studies $(3,4,8)$. 
Table 4: Histopathology diagnosis by age and sex category among cases undergone exenteration in the study.

Age $<15$ years(mean 4.1+2.89)

\begin{tabular}{lccc}
\hline Histo-pathology Diagnosis & Total & Male & Female \\
& $\mathrm{N}(\%)$ & $\mathrm{N}(\%)$ & $\mathrm{N}(\%)$ \\
\hline Retinoblastoma & $9(64.3)$ & $3(21.4)$ & $6(42.9)$ \\
Non-Hodgkin's Lymphoma & $2(14.3)$ & $2(14.3)$ & $0(0.0)$ \\
Rhabdomyosarcoma & $2(14.3)$ & $2(14.3)$ & $0(0.0)$ \\
Benign Cystic Lymphangioma & $1(7.1)$ & $1(7.1)$ & $0(0.0)$ \\
Total & $14(100.0)$ & $8(57.1)$ & $6(42.9)$ \\
\multicolumn{1}{c}{ Age $>\mathbf{1 5}$ years } & (mean 61.2+10.7) \\
\hline Histo-pathology Diagnosis & Total & Male & Female \\
\multicolumn{2}{c}{$\mathrm{N}(\%)$} & $\mathrm{N}(\%)$ & $\mathrm{N}(\%)$ \\
\hline Conjunctiva squamous cell carcinoma & $5(38.5)$ & $2(15.4)$ & $3(23.1)$ \\
Basal Cell Carcinoma & $2(15.4)$ & $1(7.7)$ & $1(7.7)$ \\
Eyelid Melanoma & $2(15.4))$ & $1(7.7)$ & $1(7.7)$ \\
Olfactory Neuroblastoma & $1(7.7)$ & $1(7.7)$ & $0(0.0)$ \\
Chroidal Melanoma & $1(7.7)$ & $1(7.7)$ & $0(0.0)$ \\
Angiosarcoma & $1(7.7)$ & $1(7.7)$ & $0(0.0)$ \\
Malignant Fibrous Histiocytoma & $1(7.7)$ & $0(0.0)$ & $1(7.1)$ \\
Total & $13(100.0)$ & $7(53.8)$ & $6(46.2)$ \\
\hline
\end{tabular}

Histopathology findings suggest retinoblastoma, $9(32.1 \%)$ as the leading cause for exenteration, which is comparable to Verma et al's report from Papua New Guinea, where retinoblastoma accounted for $40.3 \%$ of the exenterationcases (10). This finding was not consistent with the results of other studies (3-5). This might be due to differences in study area, study population, disease epidemiology and level of awareness about the disease. Retinoblastoma is a common eye and adnexal tumor that often presents late in Nepal 1113). Late presentation may be the result of lack of awareness about the disease, which ultimately necessitates exenteration to save the patient or as palliative therapy.

In addition,we found conjunctival squamous cell carcinoma, $5(17.9 \%)$, to be the second most common histopathological diagnosis requiring exenteration. This finding was similar with those noted in studies by Verma et al(10), Nemet et al(4), McKelvie et al (14) and Miller et al (15).
This study revealed total exenteration of $16(57.1 \%)$ as the most common surgical procedure followed by sub-total of 11(39.3\%) and extended exenteration of 1(3.6\%). This finding was consistent with those noted in studies by Rahman et al. and Nemet et al. $(3,5)$. The need for frequent total exenteration may be due to late presentation and the need for extensive tumor removal in our case series.

Although studies have shown that tumor free margins on histology can prevent recurrences of tumors but have little effect on long-term survival $(16,17)$, we found a large proportion of cases, $17(60.7 \%)$, with positive surgical margins. This is higher than the ones reported by Rahman et al. 29(42.0\%) (3), Ben Simon 11(32\%) (18) and Rahman et al 30 (46.9\%) (16). This difference could be due to late presentation of the patient resulting in patients with advanced disease condition excision continuing to have positive histologic margins. On the other hand, the difference in surgical protocol of each center could contribute to the variation.

The limitation of this study is its retrospective design. However,the need for exenteration in diseases where a prospective study is not feasible. 
In conclusion, retinoblastoma in children and conjunctival squamous cell carcinoma in adults were the leading causes of orbital exenteration in our study. In both cases, early detection and treatment can often salvage vision and the globe, while also preventing more severe local infiltration and metastasis. Improved patient awareness about ocular and orbital neoplasms may, in turn, lead to earlier presentation and improved patient morbidity and mortality.

\section{ACKNOWLEDGMENTS}

The authors would like to acknowledge $\mathrm{Mr}$ Dhakal and his team for their time spent acquiring patient data for this study.

\section{REFERENCES}

1. Bartisch G. Ophthalmodoulcia. Dresden 1583;3:208

2. American Academy of Ophthalmology. Basic and Clinical Science Course. Orbit, Eyelids, and Lacrimal System. Section 7, 2013-2014. San Francisco, CA, USA: American Academy of Ophthalmology; 2014.

3. Rahman I, Cook AE, Leather barrow B. Orbital exenteration: a 13 year Manchester experience. $\mathrm{Br} J$ Ophthalmol 2005; 89(10):1335-1340.

4. Duman R, Balci M, DUMAN R, Ozdogan S. Orbital Exenterations: Our experience at Ankara Oncology Research and Training Hospital. International Journal of Hematology \& Oncology/UHOD: 2013;23(3):166

5. Nemet AY, Martin P, Benger R, Kour G, Sharma V, Ghabrial R, and Danks J. Orbital Exenteration: A 15-Year Study of 38 Case. Ophthal Plast Reconstr Surg, 2007; 23(6):468-472.

6. Rose GE, Wright JE. Exenteration for benign orbital disease. $\mathrm{Br} J$ Ophthalmol, 1994; 78(1):14-8

7. Badiee P, Jafarpour Z, Alborzi A, Haddadi P, Rasuli M, Kalani M. Orbital mucormycosis in an immunocompetent individual. Iran $J$ Microbiol. 2012; 4(4):210-4.

8. Wong JC, Thampy R, Cook A. Life expectancy following orbital exenteration. $\mathrm{Br}$ J Ophthalmol. 2015; 99(1):1-4.

9. Kaur A, Khattri M, Jaiswal V. Pattern of orbital exenteration at a tertiary eye care center in North India. Nepal $J$ Ophthalmol 2012; 4(1):64-7.

10. Verma N, Murthy DP, and Kerek A. Orbital malignancy in Papua New Guinea: A 21 year review. Aust $N \quad Z \quad J$ Ophthalmol. 1999; 27(1):27-31.

11. Thakur SK, Sah SP, Lakhey M, Badhu BP. Primary malignant tumours of eye and adnexa in Eastern Nepal. Clin Experiment Ophthalmol. 2003; 31(5):415-7.

12. Badhu B, Sah SP, Thakur SK, Dulal S, Kumar $\mathrm{S}$, Sood A, et al. Clinical presentation of retinoblastoma in Eastern Nepal. Clin Experiment Ophthalmol. 2005; 33(4):386-9.

13. Saiju R, Thakur J, Karmacharya PC, Shah DN. Retinoblastoma in Nepal: a clinical profile of 30 cases. Nepal Med Coll J. 2006; $8(3): 171-5$.

14. McKelvie PA, Daniell M, McNab A, Loughnan M, Santamaria JD. Squamous cell carcinoma of the conjunctiva: a series of 26 cases. Br J Ophthalmol. 2002; 86(2):168-73.

15. Miller CV, Wolf A, Klingenstein A, Decker C, Garip A, Kampik A, et al. Clinical outcome of advanced squamous cell carcinoma of the conjunctiva. Eye (Lond). 2014; 28(8):962-7.

16. Rahman I, Maino A, Cook AE, Leather barrow B. Mortality following exenteration for malignant tumors of the orbit. $\mathrm{Br} J$ Ophthalmol. 2005; 89(11):1445-8.

17. Mouriaux F, Martinot V, Pellerin P, Patenotre P, Rouland JF, Constantinides G. Survival after malignant tumors of the orbit and periorbit treated by exenteration. Acta Ophthalmol Scand. 1999; 77(3):326-30.

18. Ben Simon GJ, Schwartz RM, Douglas R, Fiaschetti D, McCann JD, Goldberg RA. Orbital exenteration: one size does not fit all. Am J Ophthalmol 2005; 139(1): 11-17. 